\title{
Elucidation of Lipid Binding Sites on Lung Surfactant Protein A using X- ray Crystallography, Mutagenesis and Molecular Dynamics Simulations
}

Boon Chong Goh ${ }^{1}$, Huixing $\mathrm{Wu}^{2}$, Michael J. Rynkiewicz ${ }^{3}$, Klaus Schulten ${ }^{1, *}$, Barbara A. Seaton ${ }^{3}$, and Francis X. McCormack ${ }^{2, *}$

${ }^{1}$ Beckman Institute and Department of Physics, University of Illinois at Urbana-Champaign, Urbana, IL, 61801.

${ }^{2}$ Division of Pulmonary, Critical Care and Sleep Medicine, Department of Internal Medicine, The University of Cincinnati, Cincinnati, OH 45267.

${ }^{3}$ Department of Physiology and Biophysics, Boston University School of Medicine, Boston, MA 02118.

\section{Supporting information}


$\underline{\text { Index }}$

Page 3 - Figure S1. All three monomers of SP-A CRD bind to the membrane after $150 \mathrm{~ns}$.

Page 4 - Figure S2. Frequency of hydrogen bond formation between key SP-A residues and lipid.

Page 5 - Figure S3. Electrostatic potential map of lipid A and DPPC membranes.

Page 6 - Figure S4. Lateral diffusion of lipid and A and DPPC membranes.

Page 7 - Table S1. Crystallographic data and statistics.

Page 8 - Table S2. Full list of the hydrogen bond occupancy of SP-A binding.

Page 9 - Table S3. Full breakdown of interaction energies of SP-A binding.

Page 10 - Supplemental method. Calculations of lipid lateral diffusion constants. 
EPC

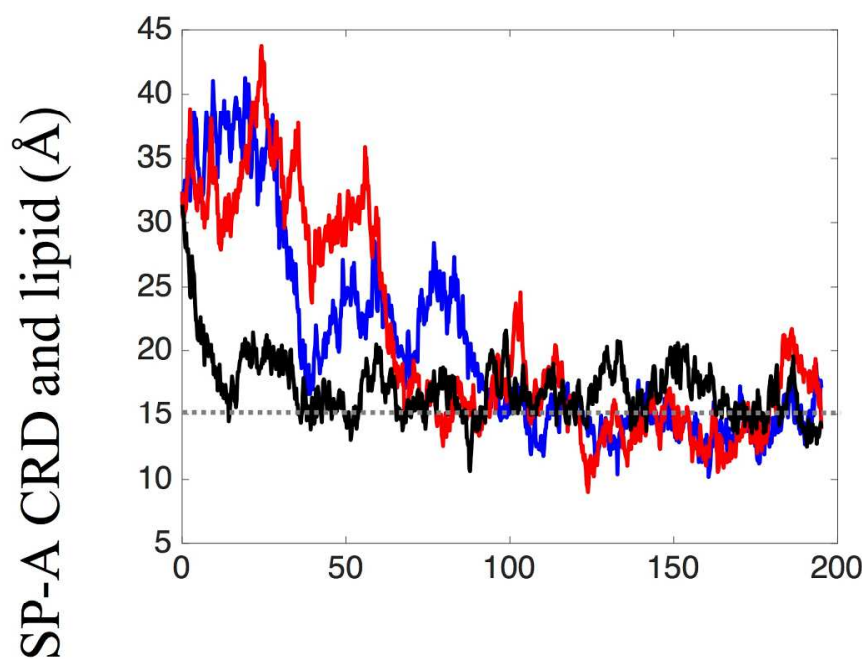

$\mathrm{EPC} 2$

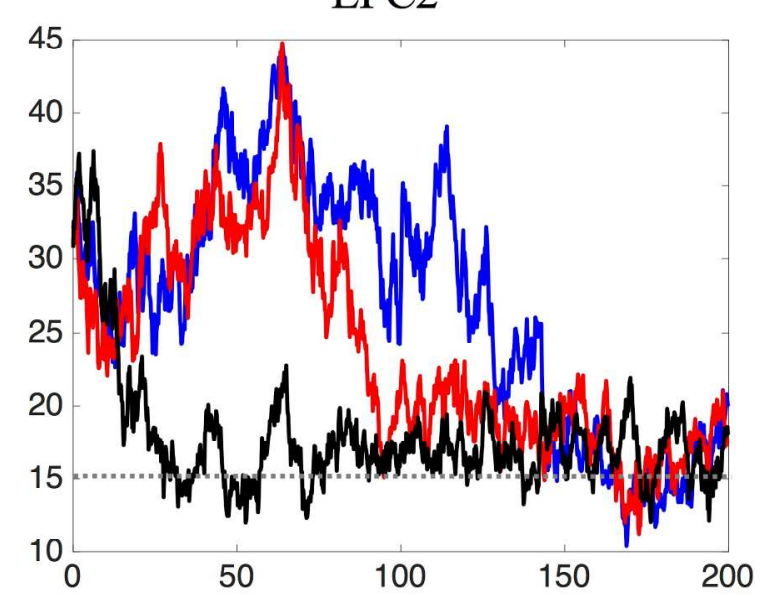

ELA

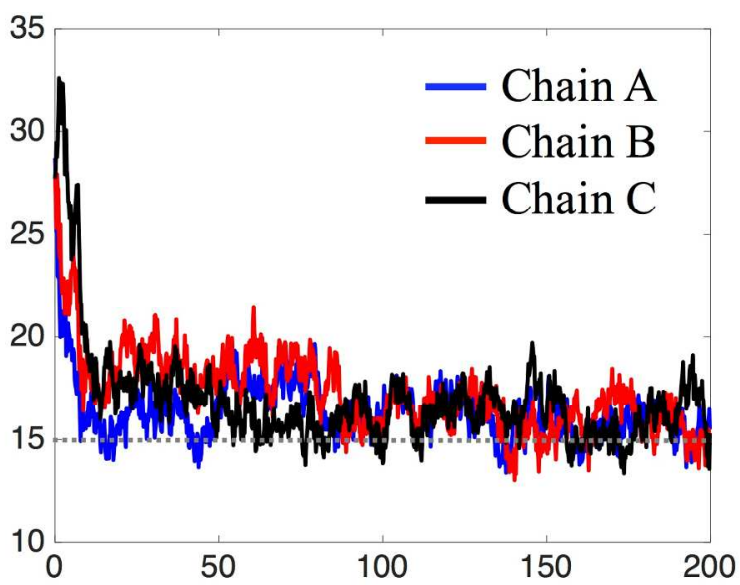

ELA2

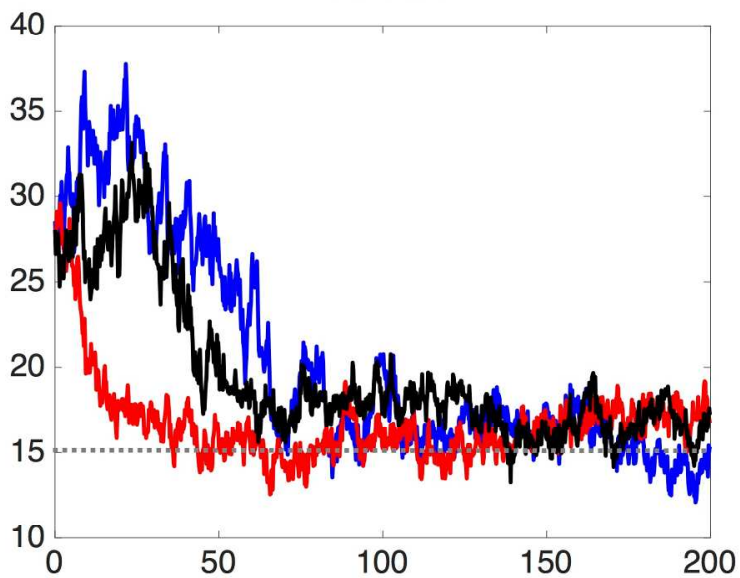

Time (ns)

Figure S1: All three monomers of SP-A CRD bind to the membrane after $150 \mathrm{~ns}$. The center of mass (C.O.M.) distances between each of the SP-A CRD monomer (residues 110 to 228) with the lipid head groups were measured for all 4 equilibrium simulations (simulations EPC, EPC2, ELA, and ELA2). Chain A of SP-A took the longest (150 ns) to establish stable binding in simulations EPC2. Note that the C.O.M. distance between a bound SP-A CRD to the membrane surface is approximately $15 \AA$ (gray dotted line). 


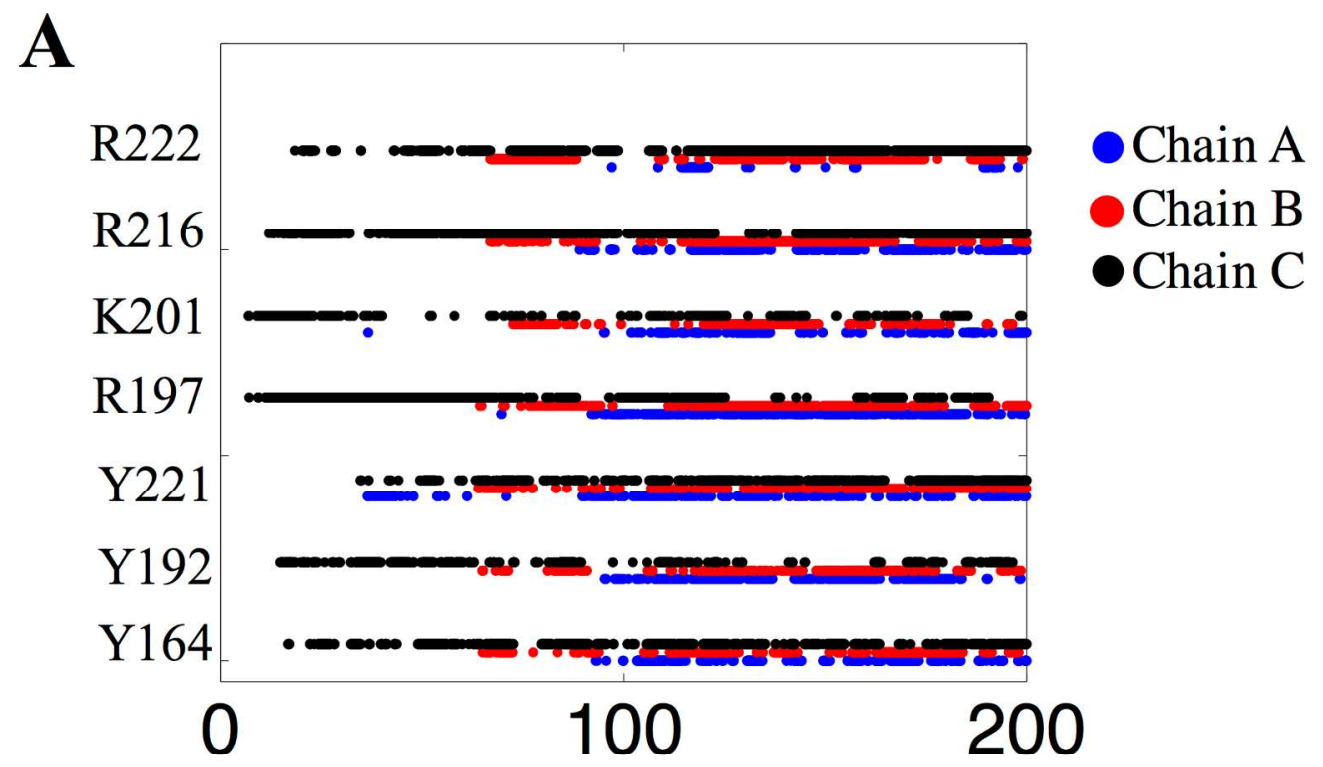

B

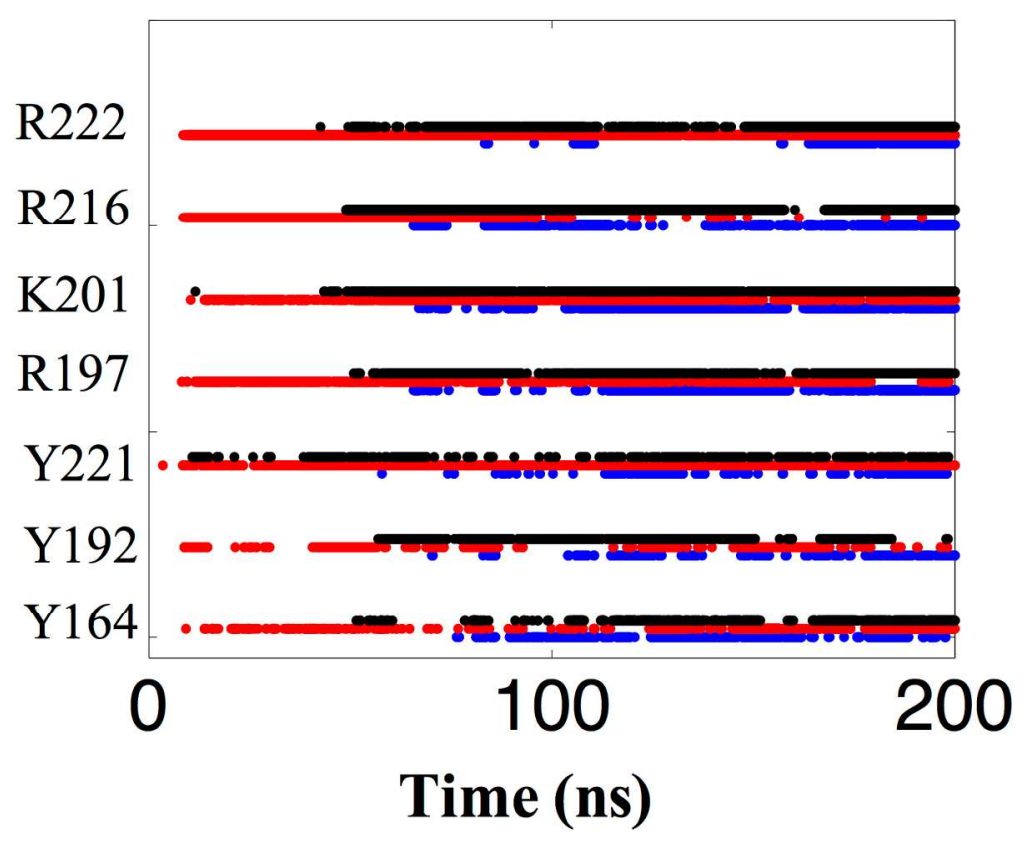

Figure S2: Frequency of hydrogen bond formation between key SP-A residues and lipid. Most residues of all three chains (blue, red and black dots) form consistent hydrogen bonds with DPPC (A) and lipid A (B) after $150 \mathrm{~ns}$ of simulation. Each point indicates a particular residue is engaged in forming hydrogen bond with the lipid membrane at a particular time. 


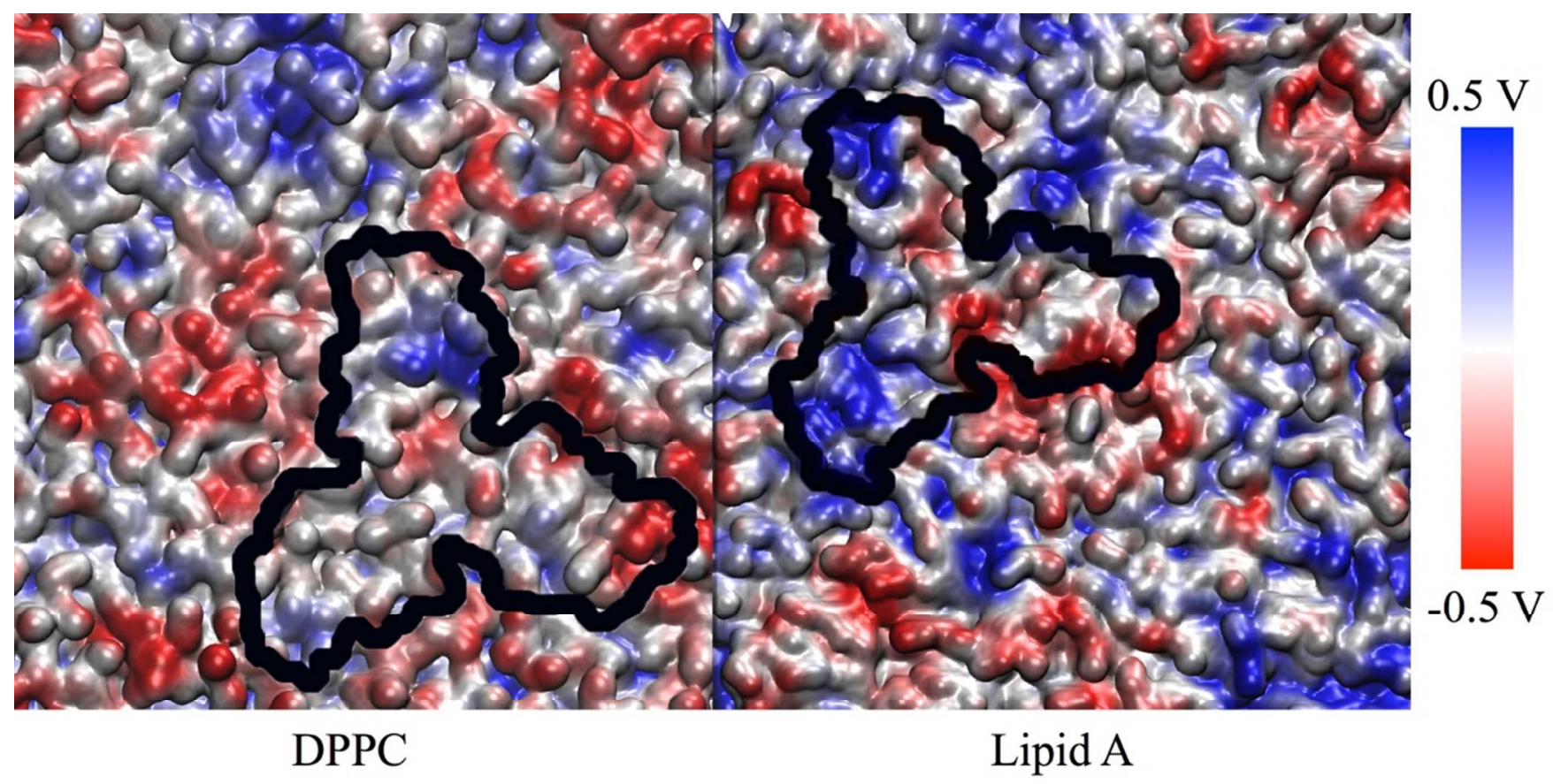

Figure S3: Electrostatic potential maps of DPPC and lipid A membranes. The membranes are colored according to their electrostatic potentials. The positions of SP-A are outlined through thick black lines. 


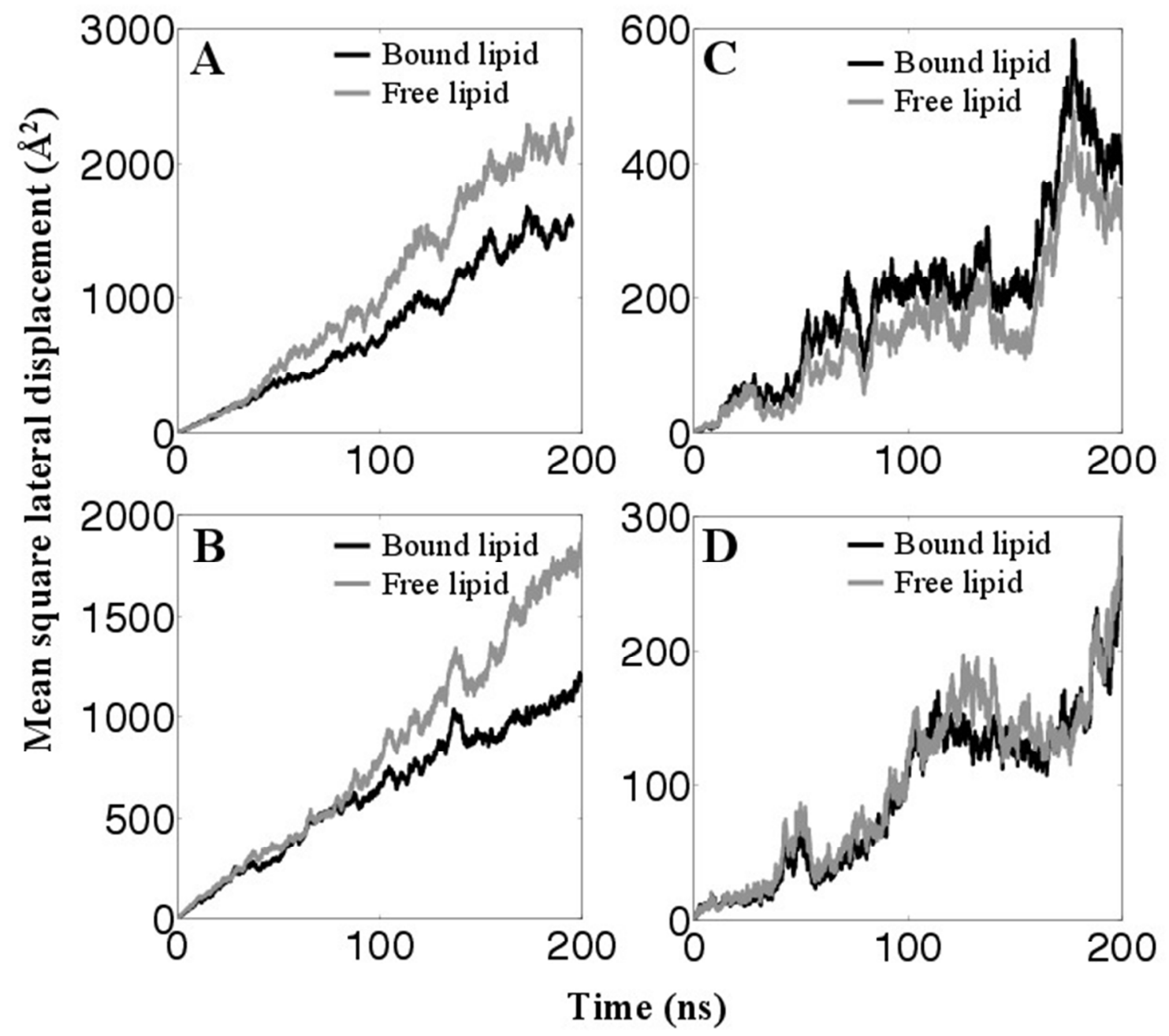

Figure S4: Lipid A diffuses seven time slower than DPPC. Implication of SP-A bound lipid to the mobility of DPPC lipid as measured from simulations SPC (Table 1). The mobility of lipid A does not seem to be affected by the binding to SP-A. The determination of the lateral diffusion constant is detailed in Supplementary Method below. 
$\underline{\text { Supplementary Tables }}$

Table S1: Crystallographic data collection and refinement statistics

\begin{tabular}{|c|c|c|c|}
\hline Construct & Wild type & Y221A & Y164A \\
\hline Ligand & phosphocholine & none & None \\
\hline PDB code & $5 \mathrm{FFR}$ & $5 \mathrm{FFT}$ & $5 \mathrm{FFS}$ \\
\hline \multicolumn{4}{|l|}{ Data Collection } \\
\hline Space group & $\mathrm{P}_{3}$ & $\mathrm{P}_{3}$ & $\mathrm{P}_{3}$ \\
\hline $\begin{array}{l}\text { Unit cell dimensions } \\
\left(\AA,^{\circ}\right)\end{array}$ & $\begin{array}{l}a=b=97.349, c= \\
44.645, \alpha=\beta=90, \\
\gamma=120\end{array}$ & $\begin{array}{l}\mathrm{a}=\mathrm{b}=97.121, \mathrm{c}= \\
44.571, \quad \alpha=\beta=90, \\
\gamma=120\end{array}$ & $\begin{array}{l}\mathrm{a}=\mathrm{b}=97.163, \mathrm{c}= \\
44.960, \alpha=\beta=90 \\
\gamma=120\end{array}$ \\
\hline Resolution $(\AA)$ & $15-2.2(2.28-2.20)$ & $90-2.0(2.07-2.00)$ & $90-1.7(1.76-1.70)$ \\
\hline No. of unique reflections & $12362(1225)$ & $16339(1624)$ & $26014(1963)$ \\
\hline Completeness $(\%)$ & $99.5(99.7)$ & $99.2(99.1)$ & $96.7(74.1)$ \\
\hline Mosaicity $\left({ }^{\circ}\right)$ & 1.587 & 0.972 & 0.373 \\
\hline Redundancy & $4.7(4.0)$ & $4.6(4.1)$ & $4.6(1.8)$ \\
\hline $\mathrm{I} / \sigma \mathrm{I}$ & $27.2(4.8)$ & $18.7(2.3)$ & $45.2(3.5)$ \\
\hline Data cutoff & $-3 \sigma$ & $-3 \sigma$ & $-3 \sigma$ \\
\hline $\mathrm{R}_{\text {merge }}$ & $0.063(0.374)$ & $0.062(0.747)$ & $0.027(0.228)$ \\
\hline \multicolumn{4}{|l|}{ Refinement } \\
\hline $\mathrm{R}_{\text {work }} / \mathrm{R}_{\text {free }}$ & $0.1693 / 0.1933$ & $0.1690 / 0.2070$ & $0.1620 / 0.1795$ \\
\hline No. of reflections & 12154 & 12074 & 22321 \\
\hline No. of atoms total & 1228 & 1258 & 1293 \\
\hline Protein atoms & 1119 & 1143 & 1154 \\
\hline Calcium and Ligands & 13 & 1 & 1 \\
\hline Waters & 96 & 114 & 138 \\
\hline \multicolumn{4}{|l|}{ Deviations from ideal } \\
\hline Bonds lengths $(\AA)$ & 0.008 & 0.005 & 0.008 \\
\hline Bond angles $\left(^{\circ}\right)$ & 0.744 & 0.714 & 1.226 \\
\hline \multicolumn{4}{|l|}{ Ramachandran plot } \\
\hline Favored $(\%)$ & 96.3 & 96.5 & 98.0 \\
\hline Allowed (\%) & 3.7 & 3.5 & 2.0 \\
\hline Outliers (\%) & 0.0 & 0.0 & 0.0 \\
\hline
\end{tabular}


Table S2: Full list of the hydrogen bond occupancy of Table 2 for all three monomers, shown in percentage. For some residues, occupancy of $>100 \%$ was observed because two residues can form more than one bond simultaneously.

\begin{tabular}{|l|ccc|ccc|ccc|ccc|}
\cline { 2 - 13 } \multicolumn{1}{c|}{} & \multicolumn{3}{c|}{ EPC1 } & \multicolumn{3}{c|}{ EPC2 } & \multicolumn{3}{c|}{ ELA } & \multicolumn{3}{c|}{ ELA2 } \\
\cline { 2 - 13 } \multicolumn{1}{c|}{} & $\mathrm{A}$ & $\mathrm{B}$ & $\mathrm{C}$ & $\mathrm{A}$ & $\mathrm{B}$ & $\mathrm{C}$ & $\mathrm{A}$ & $\mathrm{B}$ & $\mathrm{C}$ & $\mathrm{A}$ & $\mathrm{B}$ & $\mathrm{C}$ \\
\hline N162 & 15 & 17 & 13 & 12 & 17 & 36 & 9 & 3 & 40 & 13 & 14 & 27 \\
N163 & 0 & 0 & 0 & 3 & 4 & 2 & 0 & 2 & 2 & 6 & 1 & 5 \\
Y164 & 26 & 30 & 41 & 47 & 29 & 45 & 86 & 4 & 45 & 30 & 34 & 54 \\
Y192 & 45 & 37 & 23 & 52 & 9 & 20 & 16 & 0 & 66 & 14 & 17 & 16 \\
R197 & 74 & 57 & 22 & 89 & 23 & 27 & 22 & 49 & 125 & 56 & 43 & 102 \\
K201 & 14 & 19 & 15 & 32 & 42 & 30 & 43 & 175 & 115 & 68 & 36 & 101 \\
Y208 & 2 & 2 & 11 & 37 & 2 & 7 & 34 & 6 & 0 & 5 & 1 & 9 \\
R216 & 48 & 52 & 108 & 114 & 63 & 63 & 67 & 156 & 53 & 59 & 0 & 63 \\
Q220 & 12 & 34 & 65 & 50 & 55 & 31 & 6 & 73 & 45 & 38 & 39 & 34 \\
Y221 & 18 & 41 & 77 & 24 & 56 & 33 & 12 & 81 & 4 & 4 & 90 & 9 \\
R222 & 5 & 52 & 80 & 18 & 73 & 41 & 24 & 0 & 98 & 45 & 98 & 96 \\
\hline
\end{tabular}


Table S3: The breakdown of Table 2 into independent MD simulations.

\begin{tabular}{|l|rrr|rrr|rrr|rrr|}
\hline \multirow{2}{*}{$\begin{array}{l}\text { Residue } \\
\text { of SP-A }\end{array}$} & \multicolumn{10}{|c|}{ Average interaction energies (kcal/mol) } \\
\cline { 2 - 13 } & \multicolumn{1}{|c|}{ EPC } & \multicolumn{2}{|c|}{ EPC2 } & \multicolumn{3}{|c|}{ ELA } & \multicolumn{2}{c|}{ ELA2 } \\
\hline ASN162 & -5.9 & \pm & 4.6 & -6.8 & \pm & 5.1 & -5.9 & \pm & 2.5 & -7.4 & \pm & 4.8 \\
ASN163 & -3.1 & \pm & 1.5 & -3.6 & \pm & 1.7 & -2.9 & \pm & 1.4 & -2.8 & \pm & 1.7 \\
TYR164 & -5.5 & \pm & 6.1 & -5.0 & \pm & 5.7 & -8.5 & \pm & 3.4 & -6.6 & \pm & 5.0 \\
TYR192 & -7.3 & \pm & 6.8 & -8.4 & \pm & 6.9 & -4.6 & \pm & 2.4 & -3.8 & \pm & 3.4 \\
ARG197 & -17.7 & \pm & 15.8 & -25.1 & \pm & 16.4 & -20.7 & \pm & 11.8 & -22.1 & \pm & 13.2 \\
LYS201 & -7.8 & \pm & 11.7 & -4.4 & \pm & 10.2 & -38.9 & \pm & 12.4 & -21.8 & \pm & 12.5 \\
TYR208 & -3.0 & \pm & 3.9 & -2.7 & \pm & 2.2 & -3.7 & \pm & 2.7 & -1.9 & \pm & 2.1 \\
ARG216 & -26.3 & \pm & 16.4 & -33.7 & \pm & 17.4 & -35.3 & \pm & 6.5 & -18.9 & \pm & 9.0 \\
GLN220 & -12.3 & \pm & 6.6 & -15.7 & \pm & 8.6 & -10.5 & \pm & 2.9 & -10.4 & \pm & 2.8 \\
TYR221 & -8.5 & \pm & 6.4 & -10.3 & \pm & 7.7 & -10.4 & \pm & 2.9 & -9.7 & \pm & 2.9 \\
ARG222 & -17.8 & \pm & 9.3 & -27.3 & \pm & 11.8 & -16.3 & \pm & 4.3 & -28.2 & \pm & 8.2 \\
Calcium & -11.0 & \pm & 5.7 & -12.4 & \pm & 10.2 & -10.4 & \pm & 2.2 & -7.5 & \pm & 2.3 \\
\hline
\end{tabular}




\section{$\underline{\text { Supplementary Method }}$}

Lateral diffusion constants of DPPC and lipid A can be calculated as follows ${ }^{1}$

$$
\begin{aligned}
D & =\frac{M S D}{4 t} \\
& =\frac{1}{4 t N} \sum_{\forall i}\left[x_{i}(t)-x_{i}(0)\right]^{2}+\left[y_{i}(t)-y_{i}(0)\right]^{2},
\end{aligned}
$$

where MSD is the lateral mean square displacement of lipid, $t$ is total simulation time, $\mathrm{i}$ is the individual lipid molecule and $\mathrm{N}$ is the total number of lipids in the membrane.

\section{Effect of SP-A binding on DPPC and lipid A.}

SP-A-bound lipid is defined by lipid molecules that are within $30 \AA$ from SP-A.

\begin{tabular}{|l|c|}
\hline Condition of lipid & $\begin{array}{c}\text { Lateral diffusion constant, D } \\
\left(10^{-7} \mathrm{~cm}^{2} / \mathrm{s}\right)\end{array}$ \\
\hline Bound DPPC lipids & $1.8 \pm 0.4$ \\
\hline Freely-moving DPPC lipids & $2.5 \pm 0.4$ \\
\hline Bound lipid A molecules & $0.42 \pm 0.19$ \\
\hline Freely-moving lipid A molecules & $0.35 \pm 0.17$ \\
\hline
\end{tabular}

SP-A binding has a noticeable effect on the diffusion of DPPC lipids, reducing the lipid mobility by 28\%; whereas SP-A binding has no statistically significant effect on the mobility of lipid A. Additionally, judging from the freely-moving lipids, DPPC lipids diffuse laterally about seven times faster than lipid A molecules.

Reference:

1. Klauda, J. B., Brooks, B. R., and Pastor, R. W. (2006) Dynamical motions of lipids and a finite size effect in simulations of bilayers. J. Chem. Phys. 125, 144710. 